UCRL-JC-119752

PREPRINT

CONF-950420--32

\title{
Performance of a Neutron Transport Code with Full Phase Space Decomposition on the Cray Research T3D
}

RECEIVED

M. R. Dorr

OCT 061995

E. M. Salo

OSTI

This paper was prepared for submittal to

International Conference on Mathematics \& Computations,

Reactor Physics, and Environmental Analyses

Portland, Oregon, April 30 to May 4, 1995

January 1995

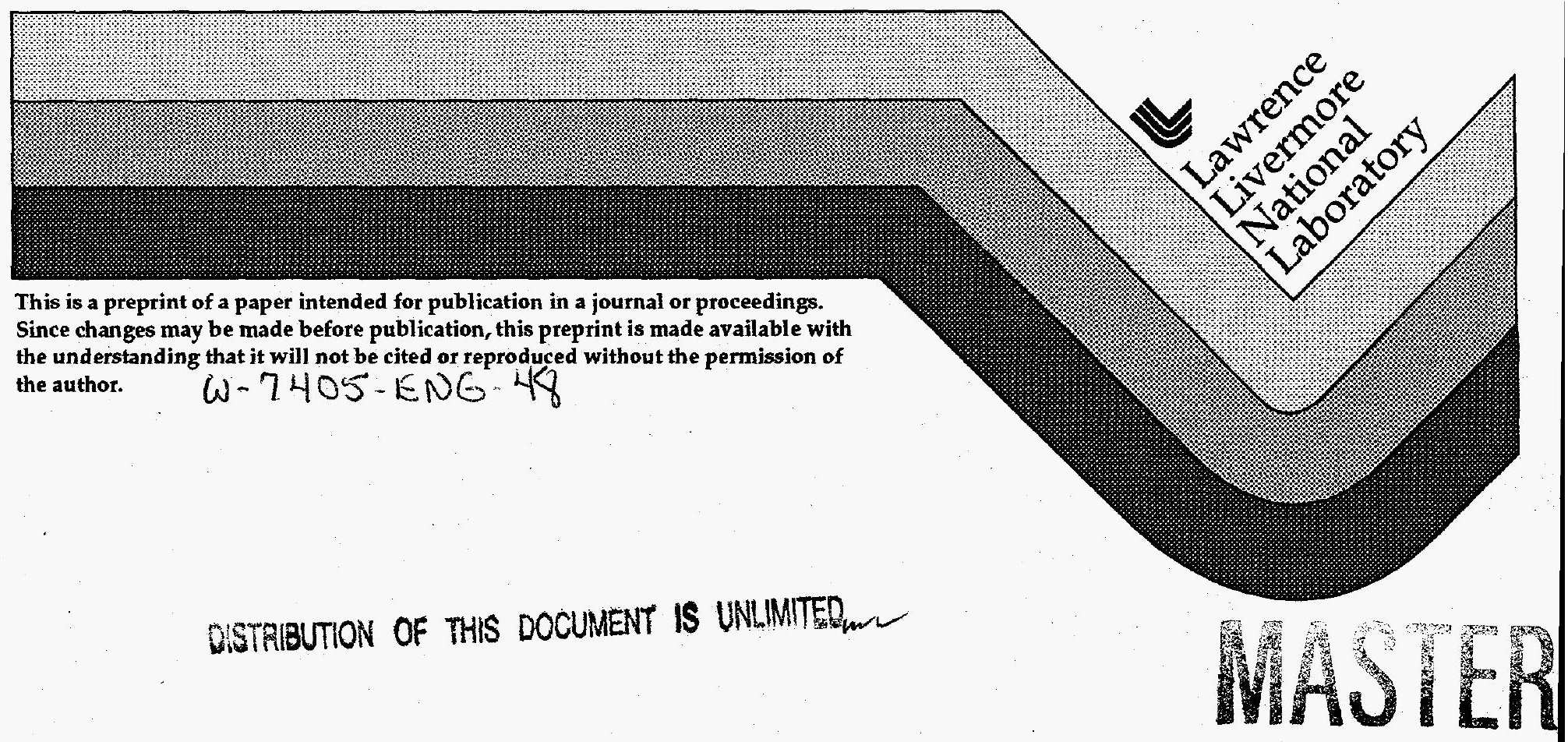




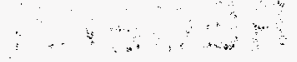

\section{DISCLAIMER}

- This document was prepared as an account of work sponsored by an agency of the United States Government. Neither the United States Government nor the University of California nor any of their employees, makes any warranty, express or implied, or assumes any legal liability or responsibility for the accuracy, completeness, or usefulness of any information, apparatus, product, or process disclosed, or represents that its use would not infringe privately owned rights. Reference herein to any specific commercial product, process, or service by trade name, trademark, manufacturer, or otherwise, does not necessarily constitute or imply its endorsement, recommendation, or favoring by the United States Government or the University of California. The views and opinions of authors expressed herein do not necessarily state or reflect those of the United States Government or the University of California, and shall not be used for advertising or product endorsement purposes. 


\section{DISCLAIMER}

Portions of this document may be illegible in electronic image products. Images are produced from the best available original document. 


\section{PERFORMANCE OF A NEUTRON TRANSPORT CODE WITH FULL PHASE SPACE DECOMPOSITION ON THE CRAY RESEARCH T3D *}

\author{
Milo R. Dorr \\ Lawrence Livermore National Laboratory \\ P.O. Box 808, L-316 \\ Livermore, CA 94551 \\ dorr@llnl.gov
}

\author{
Eric M. Salo \\ Lawrence Livermore National Laboratory \\ P.O. Box $808, \mathrm{~L}-560$ \\ Livermore, CA 94551 \\ salo@nersc.gov
}

\section{ABSTRACT}

We present performance results obtained on a 128-node Cray Research T3D computer by a neutron transport code implementing a standard multigroup, discrete ordinates algorithm on a three-dimensinal Cartesian grid. After summarizing the implementation strategy used to obtain a full decomposition of phase space (i.e., simultaneous parallelization of the neutron energy, directional and spatial variables), we investigate the scalability of the fundamental source iteration step with respect to each phase space variable. We also describe enhancements that have enabled performance rates approaching 10 gigaflops on the full 128-node machine.

1. Introduction. In [1], a strategy is presented for implementing conventional source iteration on massively parallel computers for use in the solution of multigroup, discrete ordinates neutron transport equations on threedimensional Cartesian grids. Massively parallel computers are characterized as machines comprised of hundreds or thousands of nodes (each consisting of a processor and local memory) connected by a network through which messages are passed to communicate data among the nodes. When full decompositions of phase space are considered (i.e., simultaneous parallelization of the neutron energy, direction and spatial variables), it is shown that the concurrency available in a "parallelized" version of the standard source iteration procedure can be sufficient to obtain high performance on these machines. In addition to proposing one such approach, a performance model is developed and analyzed in [1] to analytically demonstrate the scalability of the algorithm with respect to each phase space variable and to address the problem of predicting optimal phase space decompositions. The machine-specific aspects of this analysis, as well as all of the numerical results presented in [1], were obtained on a 1024-node nCUBE/2.

In this paper, we test this strategy and code on a 128-node Cray Research T3D computer. Whereas the fewyear-old nCUBE/2 used as the testbed in [1] possessed a large number of hypercube-connected nodes that could be used to exploit the high degree of concurrency in three-dimensional, multigroup, discrete ordinates calculations, each node was relatively weak. The peak processor floating point speed was just 2 megaflops, and there were only 4 megabytes of memory per node. In contrast, the newer T3D has a smaller number of much more powerful nodes arranged in a three-dimensional torus topology. The processor in each node is a DEC Alpha chip rated at 150 megaflops peak, and there are 64 megabytes of memory per node. The results reported in this paper show that on the T3D the algorithm displays the same good scalability with respect to each of the phase space variables as was demonstrated in [1] on the $\mathrm{nCUBE} / 2$. Moreover, the overall performance is an order of magnitude greater, with peak speeds approaching 10 gigaflops on the full 128-node machine.

In Section 2 we review the neutron transport equation and its discretization. A linear system is obtained whose solution by Richardson's method corresponds to conventional source iteration. The phase space decomposition strategy proposed and analyzed in [1] is summarized in Section 3. In Section 4 we discuss the implementation of the algorithm on the T3D, and in Section 5 we present some scalability and other performance results.

- This work was supported by the Applied Mathernatical Sciences subprogram of the Office of Scientific Computing, U.S. Department of Energy and by Lawrence Livermore National Laboratory under contract No. W-7405-Eng-48. 
2. Problem formulation. We consider the steady-state, neutron transport equation [2]

$$
\begin{aligned}
& \Omega \cdot \nabla \psi(x, E, \Omega)+\sigma(x, E) \psi(x, E, \Omega)= \\
& \quad \int_{0}^{\infty} \sigma\left(x, E^{\prime} \rightarrow E\right) \int_{\mathcal{S}^{2}} \psi\left(x, E^{\prime}, \Omega^{\prime}\right) d \Omega^{\prime} d E^{\prime}+q(x, E, \Omega), \quad x \in D, \quad 0<E<\infty, \quad \Omega \in \mathcal{S}^{2}, \\
& \psi(x, E, \Omega)=\psi^{i n c}(x, E, \Omega), \quad x \in \partial D, 0<E<\infty, \Omega \in \mathcal{S}^{2} \ni \Omega \cdot n_{\partial D}(x)<0
\end{aligned}
$$

where $D$ is a region in $\Re^{3}$ with boundary $\partial D, n_{\partial D}(x)$ is the outward pointing unit normal to $\partial D$ at $x$ and $\mathcal{S}^{2}$ is the unit sphere in $\Re^{3}$. Equation (1) models the transport of neutrons through an isotropically scattering background material. The unknown quantity $\psi(x, E, \Omega)$ represents the flux of neutrons at the spatial point $x$ moving in the direction $\Omega$ with energy $E$. The coefficients $\sigma(x, E)$ and $\sigma\left(x, E^{\prime} \rightarrow E\right)$ are the total and scattering cross-sections, respectively. The term $q(x, E, \Omega)$ represents an external, volumetric neutron source, while $\psi^{\text {inc }}(x, E, \Omega)$ represents a source of neutrons incident upon $\partial D$.

A semi-discretization of (1) can be obtained using a multigroup discretization of the energy $E$ and a discrete ordinates, or $S_{N}$, treatment of the neutron direction $\Omega$ (see, e.g., [3]). Using $G$ energy groups and $M$ discrete directions, one obtains a system of the form

$$
\begin{aligned}
& \Omega_{m} \cdot \nabla \psi_{g, m}(x)+\sigma_{g}(x) \psi_{g, m}(x)= \\
& \cdot \sum_{g^{\prime}=1}^{G} \sigma_{g^{\prime} \rightarrow g}(x) \sum_{m^{\prime}=1}^{M} w_{m^{\prime}} \psi_{g^{\prime}, m^{\prime}}(x)+q_{g, m}(x), \quad x \in D, \quad 1 \leq g \leq G, \quad 1 \leq m \leq M, \\
& \psi_{g, m}(x)=\psi_{g, m}^{i n c}(x), \quad x \in \partial D, \quad 1 \leq g \leq G, \quad 1 \leq m \leq M \quad \ni \Omega_{m} \cdot n_{\partial D}(x)<0 .
\end{aligned}
$$

The discretization of (1) is completed by applying a spatial discretization to (2). We assume that the spatial domain $D$ is a three-dimensional rectangular domain partitioned into a Cartesian grid of "zones". Upon this grid, any one of a variety of discretization schemes can be implemented, such as diamond-differencing, discontinuous finite element methods or modifications of these. For our present purposes, the particular choice of spatial discretization is irrelevant provided that it satisfies two conditions. First, we require that the discrete analogs of the functions $\psi_{g, m}$ can be represented by a vector $\Psi_{g, m}$ of length $N$, where $N$ is proportional to the number of zones. Letting $\Psi_{g} \equiv\left(\Psi_{g, 1}, \ldots, \Psi_{g, M}\right)^{T}$ and appropriately defining matrices and vectors $H_{g}, L, L^{+}, \Gamma_{g, g^{\prime}}, Z$, and $Q_{g}$, the fully discrete analog of $(2)$ (first divided through by $\sigma_{g}$ ) can therefore be written in matrix form as [1]

$$
H_{g} \Psi_{g}-Z L^{+} \sum_{g^{\prime}=1}^{G} \Gamma_{g, g^{\prime}} L \Psi_{g^{\prime}}=Q_{g}, \quad 1 \leq g \leq G .
$$

The second condition we require of the spatial discretization is that the zones can be enumerated (along with the corresponding entries in the matrix and vector objects in (3) in such a way that each diagonal block $H_{g, m}$ of the (block diagonal) matrix $H_{g}$ is a block lower triangular matrix such that, given any $N$-vector $v$, the product $H_{g, m}^{-1} v$ can be computed using at most $\chi_{c} N$ floating point operations storing at most $\chi_{m} N$ floating point numbers. The proportionality constants $\chi_{m}$ and $\chi_{c}$ depend upon the spatial discretization. In the common terminology of the numerical transport community, this second condition is that $H_{g, m}^{-1}$ can be applied to a vector by performing a "transport sweep" of the spatial grid in the direction $\Omega_{m}$ with a computational complexity proportional to the number of zones. Standard spatial discretizations such diamond differencing or discontinuous finite element methods satisfy both of these properties.

Equation (3) is a $G M N$-dimensional linear system. A $G N$-dimensional system is obtained by premultiplication of (3) by $L H_{g}^{-1}$, which yields, for $g=1, \ldots, G$,

$$
\Phi_{g}-K_{g} \sum_{g^{\prime}=1}^{G} \Gamma_{g, g^{\prime}} \Phi_{g^{\prime}}=L H_{g}^{-1} Q_{g}
$$

where $\Phi_{g} \equiv L \Psi_{g}$ and $K_{g} \equiv L H_{g}^{-1} Z L^{+}$. This can be written in matrix form as

$$
A \Phi=R,
$$


with $A$ and $R$ appropriately defined. By applying $H_{g}^{-1}$ to (3), we obtain

$$
\Psi_{g}=H_{g}^{-1}\left(Z L^{+} \sum_{g^{\prime}=1}^{G} \Gamma_{g, g^{\prime}} \Phi_{g^{\prime}}+Q_{g}\right)
$$

Thus, the solution of (3) can be recovered from the solution of the lower-dimensional linear system (5).

The coefficient matrix $A$ in (5) is dense and, for most practical problems, is of sufficiently large order that it is infeasible to explicitly compute and store its entries. The system (5) must therefore be solved iteratively using a method that does not require the explicit availability of $A$. Fortunately, many iterative methods satisfy this requirement, the simplest example of which is Richardson iteration, in which the recursion

$$
\Phi_{k+1}=(I-A) \Phi_{k}+R
$$

is performed until some convergence criteria is met involving the difference $\Phi_{k+1}-\Phi_{k}$, which is the residual of (5) corresponding to the approximate solution $\Phi_{k}$. In fact, the iteration (7) is precisely the well-known source iteration algorithm used in many neutron transport codes. This iteration represents the procedure of taking an initial guess of the scalar flux vector $\Phi=L \Psi$, evaluating the scattering term (involving $\Gamma$ ) as a source term for the subsequent inversion (via transport sweeps over the spatial grid) of the differential operator represented by $H$ to obtain the angular flux vector $\Psi$, which is then integrated over all directions to obtain an updated scalar flux vector $\Phi$. Higher-order alternatives to Richardson iteration, such as the adaptive Chebyshev and Generalized Minimum Residual (GMRES) methods, can also be used to solve (5) [4]. As in the simpler Richardson iteration, these methods share the common feature that the only information required about the system matrix $A$ is how to multiply it times a vector (a characteristic of Krylov subspace methods). It is also the case that this matrix-vector multiplication (source iteration) step is the dominant cost per iteration in all of these methods, excluding possible preconditioning. This fundamental kernel is therefore the focus of the parallelization approach described next.

3. Overview of the concurrent source iteration algorithm. In [1], a strategy for solving (5) on massively parallel computers was proposed and analyzed. The principal design points for the algorithm are that (i) phase space is fully decomposed (i.e., parallelized with respect to the spatial, directional and energy variables) and (ii) the wall clock time and memory utilization for a single source iteration (calculation of the matrix vector product $A \Phi$ ) scale sublinearly with respect to each of the phase space variables $G, M$ and $N$. The first of these goals ensures that all concurrency extant in the problem is exploitable, while the second criterion ansures that some benefit will be derived from applying more nodes to accommodate an increase in any or all of these phase space variables.

As described in [1], both the sequential floating point complexity and memory requirements of a single source iteration scale asymptotically like $G(G+M) N$. Hence, each of these can be modeled as a trilinear function of variables $P, Q$ and $R$ where $P$ is proportional to $G, R$ is proportional to $N$, and $Q$ is proportional to $G$ and/or $M$. This observation motivates the data layout strategy, in which one imagines that the available nodes are virtually organized as a $P \times Q \times R$ nodal grid (see Figure 1) where

- each $Q R$ node plane is assigned to a unique block of $\beta_{G}^{P}$ energy groups, which implies that $P=G / \beta_{G}^{P}$,

- each $P R$ node plane is assigned to a unique block of $\beta_{G}^{Q}$ energy groups and/or $\beta_{M}$ directions so that $Q=\max \left(Q_{G}, Q_{M}\right)$ where $Q_{G}=G / \beta_{G}^{Q}$ and $Q_{M}=M / \beta_{M}$,

- each $P Q$ node plane is assigned to a unique block of $\beta_{N}$ spatial unknowns, which implies that $R=N / \beta_{N}$. In saying that a nodal plane is "assigned" to a block of unknowns, we mean that each node of the plane contains all data corresponding to that block of unknowns and performs all operations using this data. Thus, the node with virtual coordinates $(p, q, r)$ would contain all of the entries of the angular flux array $\Psi$ corresponding to the $p$-th block of energy groups, the $q$-th block of directions and the $r$-th subdomain of spatial zones. It would also contain, for example, the entries of the scattering ratio matrix $\Gamma$ corresponding to the continuous quantities $\sigma_{g^{\prime} \rightarrow g}(x) / \sigma_{g}(x)$ with $g$ in the $p$-th block of energy groups, $g^{\prime}$ in the $q$-th block of energy groups and $x$ in the $r$-th subdomain of spatial zones. The dual role of the $Q$ axis in representing both group and directional concurrency is a consequence of our scalability requirements and the appearance of the $(G+M)$ factor in the floating point operation and memory requirement asymptotic estimates. The consequences (both favorable and unfavorable) of this fact are discussed in detail in [1]. 


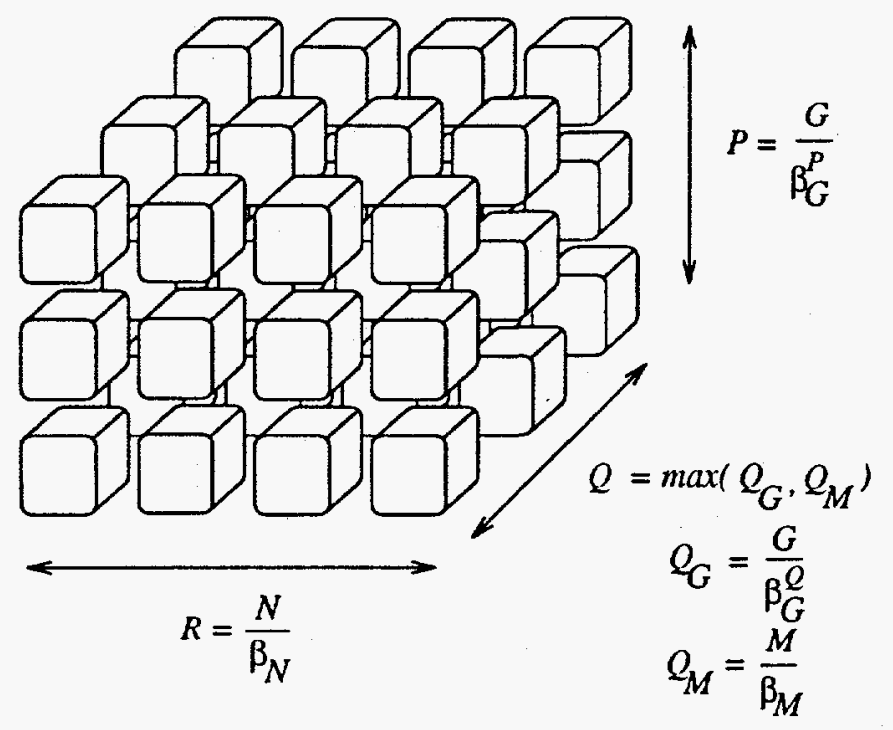

FIG. 1. Virtual $P \times Q \times R$ nodal grid.

The introduction of the $P \times Q \times R$ virtual grid also facilitates management of the communication required during a source iteration. For example, the computation of the scalar flux $\Phi$ from the angular flux $\Psi$ by numerical integration (application of the operator $L$ ) is effected by combining partial sums across the $Q$ dimension of the virtual grid, since this axis represents directional concurrency. In performing this step, only nodes with virtual grid coordinates $(p, q, r)$ with the same values of $p$ and $r$ need to communicate. Computation of the scattering term $\sum_{g^{\prime}=1}^{G} \Gamma_{g, g^{\prime}} \Phi_{g^{\prime}}$ involves global communication across both the $P$ and $Q$ dimensions, so only nodes $(p, q, r)$ with the same $r$ values participate. In neither of these two computations is any communication across the spatial variables (the $R$ dimension) required. On the other hand, the transport sweeps (application of the operators $H_{g}^{-1}$ ) do couple the spatial variables, but involve no coupling among directions or energy groups. The nodes required to perform any given transport sweep are therefore easily identified as those with virtual grid coordinates $(p, q, r)$ sharing the same values of $p$ and $q$. Using this abstract view of the machine, it is straightforward to design an independent software layer that supports communication among such subspaces of a three-dimensional nodal grid, insulating the rest of the application from the associated bookkeeping details.

The parallel execution of transport sweeps is a conceptually straightforward generalization of the sequential algorithm, but warrants some further discussion. In the sequential algorithm, for a particular neutron direction $\Omega_{m}$, incident fluxes are prescribed by known boundary conditions on three faces of the spatial domain. Fluxes in the interior of the domain are obtained by sweeping through the grid along planes that are logically perpendicular to $\Omega_{m}$. The sweeps are therefore necessarily sequential in one dimension, and parallelizable (and/or vectorizable) in the other two dimensions. The parallel implementation is obtained by replacing the role of single zones by Cartesian subdomains of zones. A sweep is therefore performed along "subdomain planes" in the direction $\Omega_{m}$ (see Figure 2). Recalling that each subdomain is assigned to a unique $P Q$ plane of nodes, within a subdomain, the angular fluxes on the three upstream (relative to $\Omega_{m}$ ) faces are computed by, and received in a message from, nodes responsible for the three subdomains sharing these faces. The subdomain grid is then swept in the direction $\Omega_{m}$, using the normal sequential algorithm, producing angular fluxes on the downstream faces, which are then sent in a message to the nodes responsible for the three subdomains sharing these faces. A node is therefore either (i) waiting for incident fluxes from three other nodes, (ii) sweeping the grid in a particular direction, starting from incident fluxes on the three upstream faces, or (iii) sending the outgoing fluxes to the three nodes responsible for the downstream subdomains. If implemented in a "reactive" manner in which nodes are allowed to cycle 
Sweep plane 1

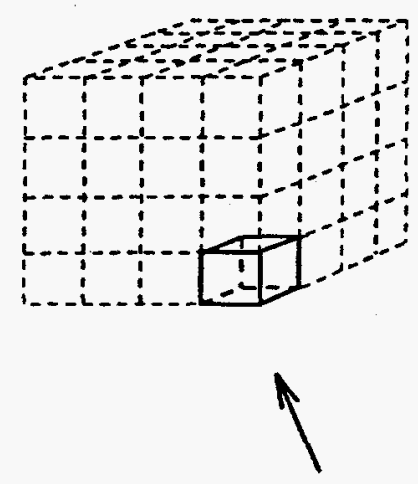

Sweep plane 2

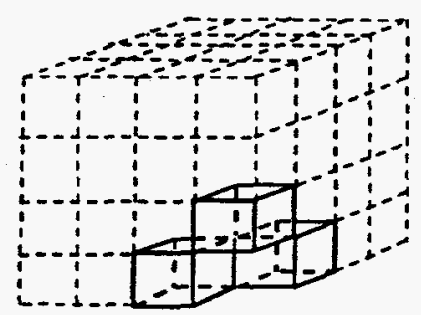

Sweep plane 3

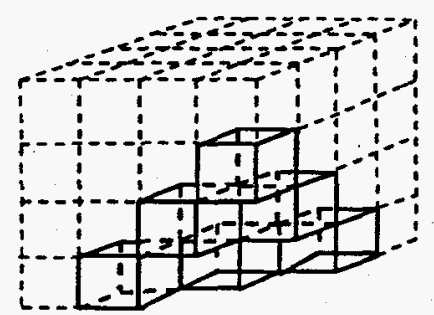

Sweep direction

FIG. 2. Grid sweeping by subdomains.

among checking for upstream fluxes, sweeping the grid and sending downstream fluxes, it is possible to avoid developing a complicated explicit scheduling algorithm by performing the computation in a purely message-driven manner. Note that, whereas communication in the $P$ and $Q$ dimensions during the formation of the scattering term involves global communication across sets of nodes, the communication in the $R$ direction during transport sweeps involves only point-to-point, communication between pairs of nodes.

Due to the sequentiality in the sweep direction, it is clear that nodes will be idle before and after the subdomain sweep plane reaches the subdomains which they contain. This limits the scalability of the wall clock time for a source iteration to a growth rate that is at best linearly dependent upon the longest dimension in the spatial grid. Nevertheless, if several directions are assigned to each $P R$ nodal plane, then multiple sweeps can be done concurrently, either by sweeping in different directions if the directions lie in distinct orthants, or by pipelined sweeps if the directions lie in the same orthant. Of course, this improvement in processor utilization has to be weighed against the fact that by assigning multiple directions to a $P R$ plane, less directional concurrency is being exploited, which could increase the wall clock time overall.

The latter point raises a very practical and important question: Given the ability to arbitrarily map phase space to machine nodes, what is the optimal decomposition for minimizing the wall clock time to perform a source iteration? This is equivalent to determining the optimal granularity of the spatial, directional and energy decompositions subject to the constraint that the total number of nodes and the amount of memory per node are fixed. In [1], a performance model is proposed that includes machine-specific parameters related to floating point speed and message-passing latency. On the 1024-node nCUBE/2 (for which reliable models of machine behavior were readily obtained), the model predicts the wall clock source iteration time for any problem and phase space decomposition to within $10 \%$ and usually much more accurately. Thus, by using this model as the objective function of a (numerically easy and inexpensive to solve) constrained minimization problem, we are able to predict a priori the optimal phase space decomposition for any given problem and number of machine nodes. Using the performance model, we are also able to analytically predict the asymptotic scalability of source iteration wall clock time with respect to each of the phase space variables. Letting $T_{\beta}\left(N_{x}, N_{y}, N_{z}, G, M\right)$ denote the wall clock time to perform a single source iteration for a problem with $G$ energy groups and $M$ directions on an $N_{x} \times N_{y} \times N_{z}$ spatial grid using a fixed phase space decomposition $\beta=\left(\beta_{N}, \beta_{G}^{P}, \beta_{G}^{Q}, \beta_{M}\right)$ (which determines the growth in the number of machine nodes resulting from an increase in the phase space dimensions), we obtained the following scaled speedup estimates:

$$
\begin{aligned}
& T_{\beta}\left(N_{x}, N_{y}, N_{z}, G, M\right)=O\left(\max \left(N_{x}, N_{y}, N_{z}\right)\right) \text { as } N=N_{x} N_{y} N_{z} \rightarrow \infty, \\
& T_{\beta}\left(N_{x}, N_{y}, N_{z}, G, M\right)=O\left(\log _{2} G\right) \text { as } G \rightarrow \infty \\
& T_{\beta}\left(N_{x}, N_{y}, N_{z}, G, M\right)=O\left(\log _{2} M\right) \text { as } M \rightarrow \infty
\end{aligned}
$$

The logarithmic growth in (9) and (10) is due to communication costs, whereas the growth estimate in (8) reflects 
the consequences of sequential transport sweeps, as discussed above. Nevertheless, this parallelization of the sequential algorithm produces scaled speedups that are sublinear in each of the phase space dimensions $G, M$ and $N$ (assuming the aspect ratio of the spatial domain is bounded as $N$ is increased).

4. T3D implementation. The code created to implement the algorithm summarized in the preceding section is called Ardra. It is written in the C programming language to exploit support for data structures and standardized dynamic memory management. Ardra currently uses diamond-difference spatial discretization, and sufficient generality has been included to facilitate future incorporation of other discretizations. The data layout and communication functions required to support the $P \times Q \times R$ virtual machine abstraction described in Section 3 are contained in a modular software layer that isolates most of the code from machine-specific implementation details. This communication layer has message-passing semantics that define specific functionality required on target machines. As a result of porting this layer to the Zipcode message passing system [5], Ardra runs on Sun Sparcstation clusters, the $\mathrm{nCUBE} / 2$ and the Intel Paragon. By implementing the layer atop the Elan Widget Library, Ardra has also been ported to the Meiko CS-2 computer. For the latter machine, it was also necessary to restructure the transport sweep routine to take advantage of the vector processors.

The port of Ardra to the T3D was accomplished by implementing the communication layer atop the Cray Research Shared Memory (SHMEM) library. At the heart of this library are functions that allow a node to "put" data into the address space of another node, or "get" data from the address space of another node. Various collective communication operations (reductions, barriers, etc.) can also be defined on groups of nodes, which facilitated the implementation of the abstract $P \times Q \times R$ nodal grid.

Using the Cray Research Apprentice profiling tool, it was determined that the Ardra port obtained in this manner spent at most $10 \%$ of its total run time in communication, and the computationally intensive transport sweeps were running at a rate of about 30 megaflops in each node. Given the $150 \mathrm{MHz}$ Alpha chip clock speed, we believed that it should be possible to boost the single node performance further. Detailed investigations revealed opportunities for additional speedups by rearranging data to increase cache locality and prefetching data for subsequent loop iterations in order to reduce the impact of cache misses. In trying to coerce the compiler to exploit some of these opportunities, the resulting source code became increasingly more assembly-like, and still did not produce the performance we had hoped to achieve. We therefore decided to rewrite the transport sweep routine directly in Alpha assembly language. The details of the assembly implementation are beyond the scope of this brief article, but the net result was that an approximate factor of three (3) speedup was gained over the compiled version. Using assembly-coded transport sweeps, we achieved speeds as high as $\mathbf{8 7}$ megaflops per node, although the actual rate for a given problem depends upon the relative amount of time spent in the optimized sweep routine, as shown in the following section.

5. Numerical results. Using Ardra, we examined the scalability of our algorithm and the performance of the code on a 128-node T3D. All of the results reported below are for the computation of a single source iteration, i.e., the computation of $A \Phi$ using the algorithm described in Section 3. The cost of this operation will dominate the total cost per iteration of solving (5) using standard iterative methods. Therefore, in these tests the numerical values of problem-dependent quantities such as $\Gamma$ or the physical dimensions of the spatial domain are irrelevant, since the values of these variables only affect the number of iterations required, not the cost per iteration. The latter cost is determined entirely by the number of groups $G$, the number of directions $M$, and the number of spatial unknowns $N$, i.e., the phase space dimensions.

In reporting the number of floating point operations required to form $A \Phi$, we count only sequential operations. That is, we only count the number of floating point operations that would have been required had the operation been performed on a sequential machine. This number could be substantially less than the number of floating point operations actually performed by the machine. For example, in performing a combine operation with a recursive doubling algorithm, scalability is achieved by performing some redundant computation to reduce communication. However, we do not count such redundancies in our floating point operation count. To do so would be to disguise communication costs as useful floating point work.

The times reported for a single source iteration (denoted by "SI Time" on graph ordinates) is the average of the times to compute $A \Phi$ in ten successive source iterations. On the T3D, the variation of times from iteration to iteration was negligible, so there was no need to compute, for example, a standard deviation. Also, all times reported are for the version of the code containing the Alpha assembly-coded transport sweep routine. 


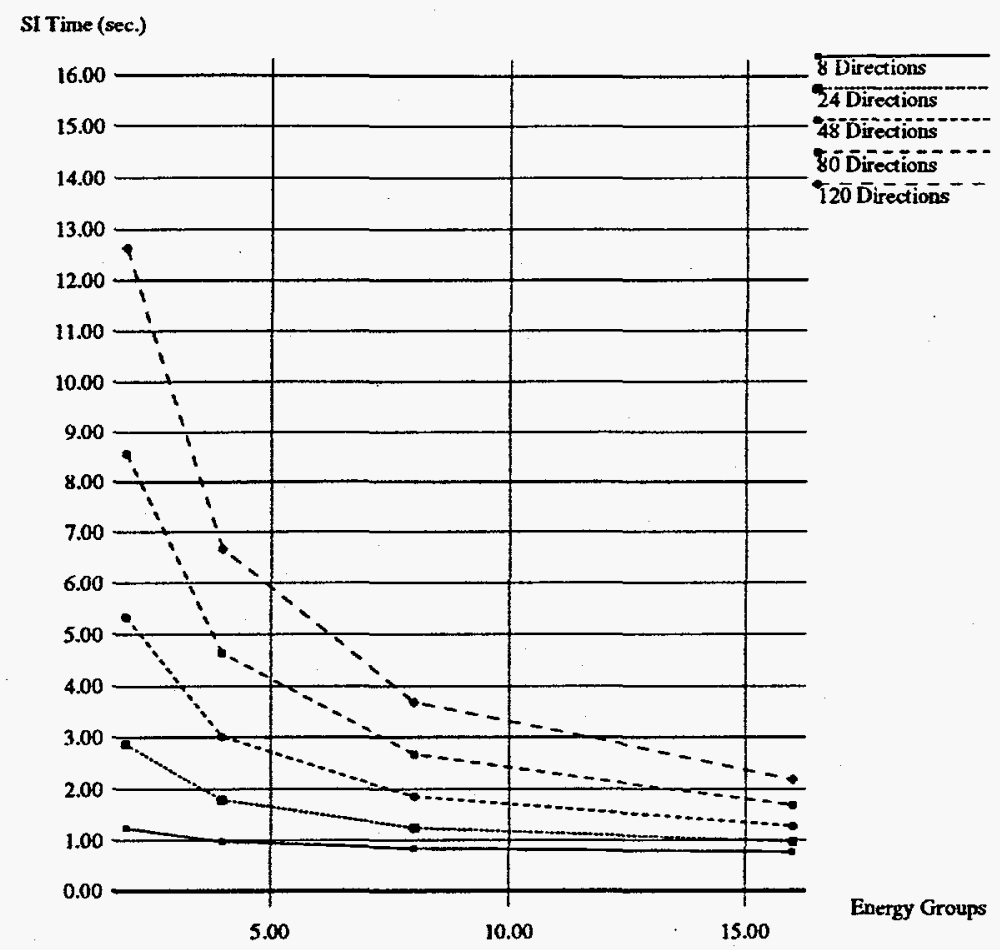

FIG. 3. Scalability of source iteration time with respect to an increasing number of energy groups.

5.1. Scalability in energy. Our first set of problems tests the scalability of the algorithm with respect to the number of energy groups $G$. Specifically, the number of directions $M$ and spatial unknowns are held fixed while the number of groups $G$ and the number of nodes $\rho$ are increased. The spatial domain is a $60 \times 60 \times 60$ cube, and no spatial decomposition is used (i.e., $N=216000$ and $R=1$ ). In all cases, $\beta_{G}^{P}=1$, assigning one energy group to each nodal grid $Q R$ plane, $\beta_{G}^{Q}=2$, assigning two energy groups to each $P R$ plane, and the blocking factor $\beta_{M}$ is chosen to be the smallest divisor of $M$ satisfying $M / \beta_{M} \leq G$.

Figure 3 displays the results for this test suite. Each of the five curves shown corresponds to a set of runs with $M$ fixed at $8,24,48,80$, or 120 while $G$ varies over $2,4,8$ or 16 . In all five cases, the source iteration time decreases as the number of groups $G$ and the number of nodes $\rho=P Q=\frac{1}{2} G^{2}$ is increased. Although one would expect a scaled speedup curve such as this to be at best. constant or logarithmically growing, the decrease in time is due to the fact that by increasing $Q$ to accommodate an increasing number of groups $G$, more directional concurrency is also being exploited (recall the dual role of the $Q$ axis in the nodal grid). The last data point on each curve corresponds to $\rho=128$, which is the maximum number of nodes available on the T3D. Since $Q=G / 2=8$ at this data point, the $P R$ nodal grid planes exhaust direction concurrency. Beyond this point (i.e., for larger values of $G$ and $\rho$ ), the number of $P R$ nodal grid planes exceeds direction concurrency, so the source iteration time would become more nearly constant or grow logarithmically as predicted by (9). This behavior was, in fact, observed on the 1024-node $\mathrm{nCUBE} / 2$ [1].

5.2. Scalability in direction. Our second set of problems tests the scalability of the algorithm with respect to the number of directions $M$, holding the number of energy groups $G$ and the number of spatial unknowns $N$ fixed. For all problems, we set $\beta_{G}^{P}=\beta_{G}^{Q}=1$ (one energy group per $Q R$ or $P R$ nodal grid plane) and $\beta_{M}=1$ (one direction per $P R$ nodal grid plane). The spatial domain is again a $60 \times 60 \times 60$ cube, and no spatial decomposition is used (i.c., $N=216000$ and $R=1$ ). Figure 4 displays the resulting timing measurements. Each curve was generated by fixing the number of groups at $G=1,2$ or 4 while increasing the number of directions $M$ and number of nodes $\rho=P Q=G M$. Figure 4 shows that the source iteration time increases only very slowly in the 


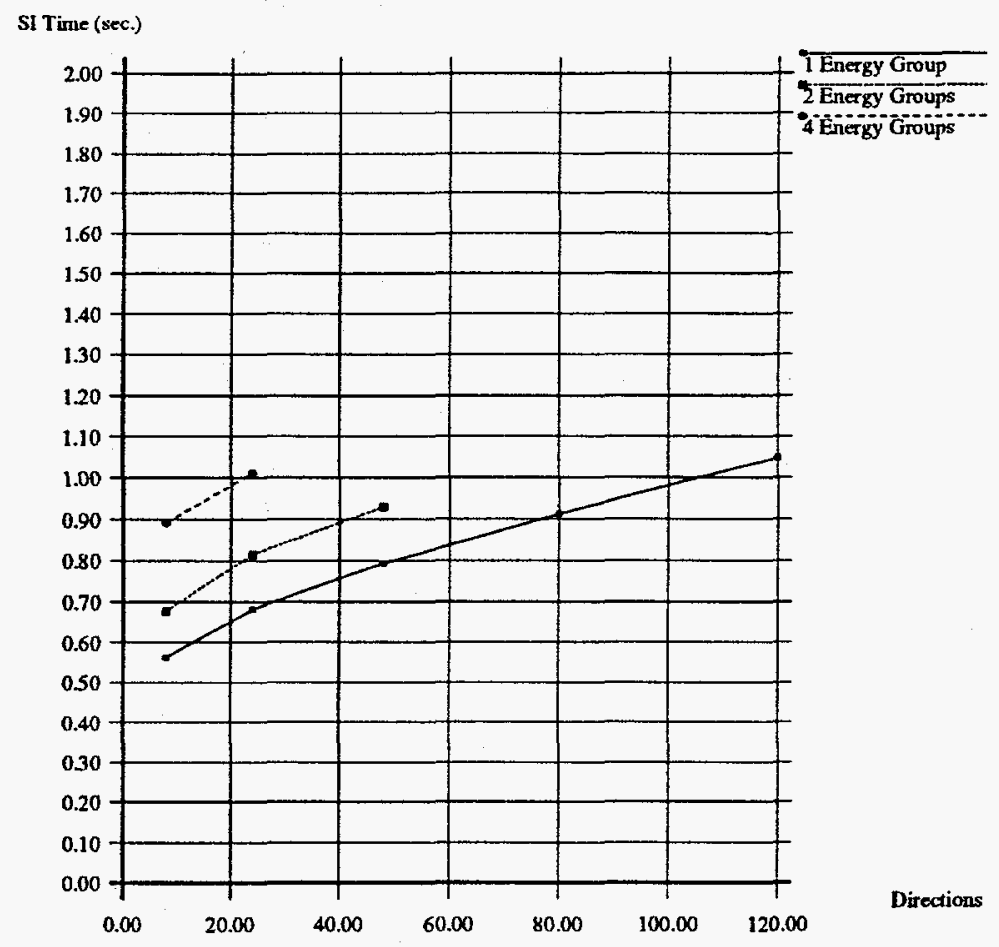

FIG. 4. Scalability of source iteration time with respect to an increasing number of directions.

\begin{tabular}{c|cccccc} 
Problem Set. & $G$ & $M$ & $\beta_{G}^{P}$ & $\beta_{G}^{Q}$ & $\beta_{M}$ & $\beta_{N}$ \\
\hline 1 & 1 & 8 & 1 & 1 & 8 & 216000 \\
2 & 2 & 8 & 1 & 2 & 8 & 216000 \\
3 & 4 & 8 & 1 & 2 & 4 & 216000 \\
4 & 8 & 8 & 1 & 2 & 2 & 216000
\end{tabular}

Problem set parameters for spatial scalability tests.

limit of large $M$, as would be predicted by (10). Note, for example, that for the $G=1$ problem the time barely doubled in going from 8 to 120 directions. It was not possible to extend the other two curves any further, due to the lack of additional nodes. Based on the analogous nCUBE/ 2 results in [1], we would nevertheless expect similar behavior of these curves on a larger machine.

5.3. Scalability in space. Our third suite of problems tests scalability with respect to the number of spatial subdomains $R$. For each of the four problem sets in this suite, the number of groups $G$, directions $M$ and the blocking factors $\beta$ are held fixed at the values shown in Table 1 , while $R$ is increased. Each subdomain consists of a $60 \times 60 \times 60$ array of zones, and the number of such subdomains is increased by alternately adding a subdomain in each coordinate direction until no further nodes are available. Thus, we begin with a single subdomain $(R=1)$, considered as a $1 \times 1 \times 1$ "subdomain array". We then increase $R$ by successively adding subdomains in the $x$ and $y$ and $z$ directions to obtain $2 \times 1 \times 1(R=2), 2 \times 2 \times 1(R=4), 2 \times 2 \times 2(R=8), 3 \times 2 \times 2(R=12)$, $3 \times 3 \times 2(R=18), 3 \times 3 \times 3(R=27)$ subdomain arrays, etc.

The results shown in Figure 5 indicate a fairly slow rate of increase in the source iteration time as the number of subdomains $R$ (and therefore the total number of spatial unknowns) and number of nodes $\rho$ are increased. From (8), we would expect an $R^{1 / 3}$ growth rate (since $N_{x}, N_{y}$ and $N_{z}$ are all approximately proportional to $R^{1 / 3}$ ). In Figure 5, we therefore plot the function $0.75 R^{1 / 3}$ for comparison. 


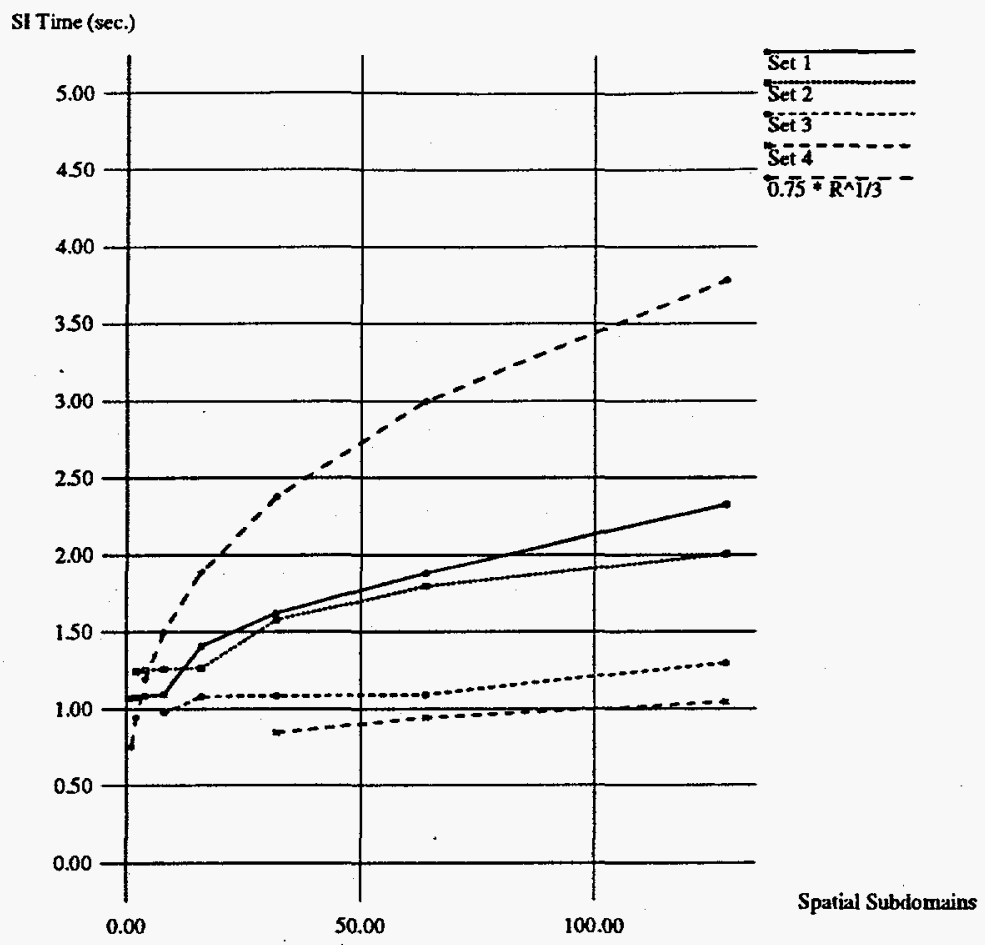

FIG. 5. Scalability of source iteration time with respect to an increasing number of subdomains.

5.4. Other results. By reviewing the steps involved in a source iteration, it is clear that the majority of floating operations are performed in transport sweeps. Our final set of numerical results show that, at least on the T3D with its DEC Alpha chip, the more time that a node spends doing transport sweeps relative to other calculations required for source iteration, the higher the floating point performance will be. Although this observation is somewhat more pronounced using the Alpha assembly-coded version of the transport sweep routine than with the compiled version (as one would expect), the main reason behind this is the apparent fact that the transport sweeps have more favorable memory access patterns and density of floating point operations than the other parts of the source iteration procedure, which are comprised largely of common vector operations.

For all problems in this set, $G=16, \beta_{G}^{P}=1, \beta_{G}^{Q}=2$ and the number of nodes $\rho=128$. The spatial domain is again a $60 \times 60 \times 60$ cube and with no decomposition (i.e., $N=216000$ and $R=1$ ). The total number of directions $M$ and the blocking factor $\beta_{M}=M / 8$ are both increased, thereby increasing the work per node solely by increasing the number of transport sweeps per node. In Figure 6 , we display the floating point rate (in gigaflops) as a function of the number of transport sweeps performed in each node during a single source iteration. The curve appears to be tending toward an asymptote slightly above 11 gigaflops. We note, however, that this level of performance corresponds to problems with several hundred discrete directions, which is a much higher level of discretization than many problems require. It is also observed that the floating point rate increases rapidly in going from a single direction per node to, say, 20 directions per node. For example, the floating point rate using 3 directions per node is approximately 2.3 times the floating point rate using just 1 direction per node. This implies that, even if the number of machine nodes were tripled and all of the extra nodes were applied to reduce the granularity of the direction variable from 3 directions per node to just 1 , the factor of 2.3 drop in average single node floating point performance would nullify about $75 \%$ of the expected speedup in wall clock time. This rough analysis ignores communication effects, but other data we have obtained indicates that this is not a significant factor. For this reason, it may not be as beneficial to parallelize over directions relative to other phase space variables, in spite of the fact that parallelization over directions would a priori seem to be an "obvious" good thing to do. The complexity of these kinds of issues argues for the further modification of the 


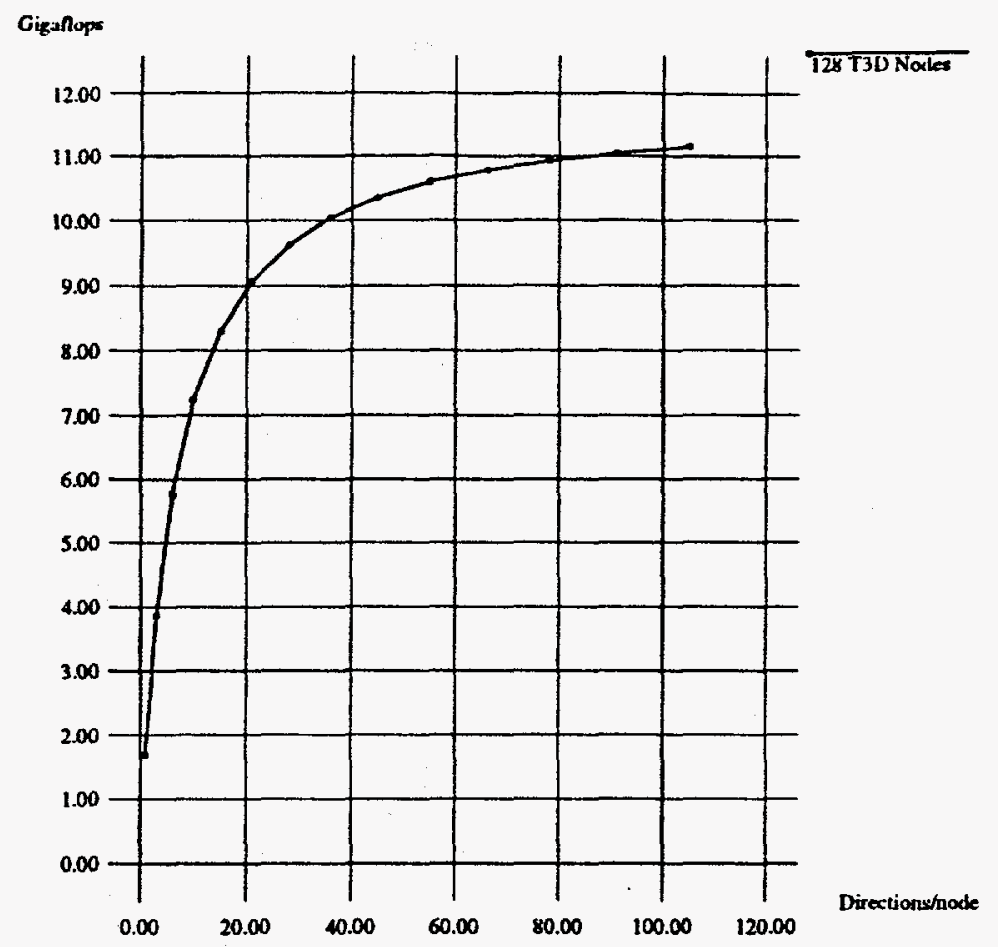

Fig. 6. Dependence of flop rate on number of directions per node.

performance model developed in [1] for the nCUBE/2 to apply to the T3D. This is a topic for future research.

Acknowledgements. The Cray Research T3D experiments described in this paper were run on the 128node machine located in the Center for Computational Sciences and Engineering at Lawrence Livermore National Laboratory as part of the High Performance Parallel Processing Project (H4P) technology transfer initiative funded by DOE Defense Programs.

\section{REFERENCES}

[1] M. R. Dorr and C. H. Still, "Concurrent Source Iteration in the Solution of Three-Dimensional, Multigroup, Discrete Ordinates Neutron Transport Equations", Lawrence Livermore National Laboratory Report UCRL-JC-116694, June 1994, subınitted to Nucl. Sci. Eng.

[2] G. I. Bell and S. Glasstone, Nuclear Reactor Theory, Van Nostrand, New York, 1970.

[3] G. I. Marchuk and V. I. Lebedev, Numerical Methods in the Theory of Neutron Trensport, Harwood Academic Publishers, Chur, 1986.

[4] S. F. Ashby, P. N. Brown, M. R. Dorr and A. C. Hindmarsh, "Preconditioned Iterative Methods for Discretized Transport Equations", in Proc. International Topical Meeting of the American Nuclear Society-Advances in Mathemetics, Computations, and Reactor Physics, Volune 2, American Nuclear Society, April 1991, pp. 6.1 2-1 to 6.1 2-9. Also Lawrence Livernore National Laboratory Teduical Report UCRL-JC-104901, July 1990.

[5] A. Skjellum and M. Morari, "Zjpcode: A Portable Communication Layer for High Performance Multicomputing-Practice and Experience", Tech. Report UCRL-JC-106725, Lawrence Livenuore National Laboratory, March 1991. To appear in Concurrency: Practice \& Experience. 